\title{
Clinical and Molecular Evaluation of a Case of Male Infertility and Azoospermia
}

Colao $E^{1}$, Vismara MFM ${ }^{1,3,4}$, Bombardiere $\mathrm{F}^{1,3}$, Fabiani $\mathrm{F}^{1}$, Grillone $\mathrm{T}^{1,3}$, Iuliano $\mathrm{R}^{1,3}$, Luciano $\mathrm{E}^{1,3}$, Nocera $\mathrm{D}^{1,3}$, Nucara $\mathrm{S}^{1}$, Primerano $\mathrm{A}^{1,3}$, Tallerico $\mathrm{R}^{1,3}$, Trapasso $\mathrm{F}^{1,3}$, Simonetta $\mathrm{M}^{1}$, Taverna $\mathrm{D}^{1,3}$, Villella $\mathrm{C}^{1}$, Perrotti $\mathrm{N}^{* 1,2,3}$ and Malatesta $\mathrm{P}^{* 1}$

${ }^{1}$ Unità Operativa Complessa di Genetica Medica, Università "Magna Graecia” di Catanzaro, Catanzaro, Italy

${ }^{2}$ Dipartimento di Scienze della Salute, Università "Magna Graecia” di Catanzaro, Catanzaro, Italy

${ }^{3}$ Scuola di Specializzazione in Genetica Medica, Università “Tor Vergata” di Roma, Italy

${ }^{4}$ Dipartimento di Medicina Molecolare, "Sapienza" Università di Roma, Italy

${ }^{*}$ Corresponding author: Malatesta P and Perrotti N, A.O.U. Mater Domini via Tommaso Campanella 115 88100 Catanzaro, Italy. Building A, 2 ${ }^{\text {nd }}$ floor, E-mail: paolissima2@yahoo.it; perrotti@unicz.it

Citation: Colao E, Vismara MFM, Bombardiere F, Fabiani F, Grillone T, et al. (2015) Clinical and Molecular Evaluation of a Case of Male Infertility and Azoospermia. J Case Rep Stud 3(1): 102. doi: 10.15744/23489820.2.402

Received Date: October 30, 2014 Accepted Date: January 30, 2015 Published Date: February 03, 2015

\section{Abstract}

Here we describe the case of a 35 year old male, with a harmonic phenotype, who sought medical assistance to assess the etiology of his infertility. As a result of clinical examination, karyotyping, FISH and molecular studies, we obtained the following karyotype: mos 45,X[18]/46,XY,idic $(Y) q(11.2)[82]$. Furthermore, the $Y$ chromosome was characterized by the absence of the regions AZF2 (former $\mathrm{AZFb}$, within the 11.22 band, and AZFc, within the 11.23 band), whereas the region AZF1 was conserved. Male infertility can be caused by several genetic alterations. Y chromosome aberrations are very frequent in infertile males. This case with an azoospermic patient carrying a complex karyotype demonstrated that cytogenetic and molecular profiling can be helpful to establish a diagnosis in cases of subjects with a normal gross phenotype.

Keywords: Mosaicisms; Fertility; Sterility; Cytogenetics; FISH; 45,X cell line; Isodicentric Y chromosome

\section{Introduction}

$\mathrm{Y}$ chromosome infertility is characterized by azoospermia (absence of sperm), severe oligozoospermia $\left(<1 \mathrm{x} 10^{6} \mathrm{sperm} /\right.$ $\mathrm{mL}$ semen), moderate oligozoospermia (1-5 x $10^{6} \mathrm{sperm} / \mathrm{mL}$ semen), or mild oligozoospermia $\left(5-20 \times 10^{6} \mathrm{sperm} / \mathrm{mL}\right.$ semen) [1]. Detection of Y chromosome microdeletion is valuable in the treatment of patients with nonobstructive azoospermia and oligoasthenoteratozoospermia [2]. Microdeletions in the Y chromosome are associated with oligo-azoospermia. The frequency of microdeletions in the AZF regions (1 and 2), as well as in the DAZ1/DAZ2 genes ranges from $0.9 \%$ to $2 \%$ in the Italian population with severe oligospermia (less that $5 \times 10^{6}$ sperm per $\mathrm{ml}$ ) [3]. Although a partial microdeletions in the AZFc region of the Y chromosome can occur in men with impaired as well as normal spermatogenesis [4]. In many cases an isodicentric Y chromosome has been described as part of a mosaic that includes a 45,X cell line [5]. Despite the identification of the genes included in the $\mathrm{Y}$ chromosome and the detailed molecular knowledge of the regions that control spermatogenesis [6], it is not yet clear whether the sterility observed in subjects with isodicentric Y chromosome can be attributed to chromosomal rearrangement that may interfere with the correct pairing of the heterochromosomes, or to contemporary deletion of the AZF regions which are important in spermatogenesis [3,7]. The Y chromosome is important for male development as it contains the sex determining gene SRY and many spermatogenesis genes, like AZF1 and 2, DAZ, etc.

\section{Case report}

Data refers to a 35 years old male subject who reported to this Unit because of infertility. He had a harmonic phenotype and no abnormalities were found during the physical examination, external genitalia G5-PH5 according to Tanner's classification, prostate gland examination was normal; height $172 \mathrm{~cm}$, weight $68.5 \mathrm{Kg}$. LH and testosterone were tested twice and were always within normal limits, whereas FSH was increased as is always the case in primary testicular defects. A sperm count revealed normal volume, but without cells as in azoospermia. A biopsy of the testes showed the presence of round spermatides. Testes ultrasound resulted in vas deferens agenesia (right) and obstruction of the vas deferens (left) (screening of 56 common mutations in CTFR genegave no positive results). Cardiac and renal ultrasonographies were normal. At the time of birth the mesaures were height $48 \mathrm{~cm}\left(10^{\circ}-25^{\circ}\right.$ pc), weight $3250 \mathrm{~g}\left(25^{\circ}-50^{\circ} \mathrm{pc}\right)$, cranial circumference $34.5 \mathrm{~cm}\left(25^{\circ}-50^{\circ} \mathrm{pc}\right)$, Apgar score $10 / 10$, neither lymphedema nor jaundice were reported. A standard karyotype was performed. Chromosomes from peripheral blood lymphocytes were analyzed. A sample of 100 mitosis were analyzed by GTG banding, with 550 bands resolution, resulting in a mosaicism 45,X[18]/46,XY,del(Y)(q11.2) [82]. The Y chromosome, when present, appeared smaller than normal. QFQ banding by fluorescent microscopy was normal, it just revealed the absence of the heterochromatic region on the long arm of the Y chromosome, a normal polymorphism feature. 
The finding was confirmed by CBG banding (Figure 1) suggesting the opportunity to better characterize the Y chromosome by Fluorescent In Situ Hybridization (FISH). For the FISH analysis of the Y chromosome, the following probes were used:

- Alpha satellite probe specific for a region including the centromere and spanning from Yp11.1 to Yq11.1 (Vysis, USA)

- LSI SRY probe, specific for the SRY gene on the short arm of the Y chromosome (Yp11.3) (Olimpus, USA)

- Multi-color DNA probe mixtures were used to identify the subtelomeric regions from the short (Yp) and the long arm (Yq) of the Y chromosome (ToTelVysion, USA).

a

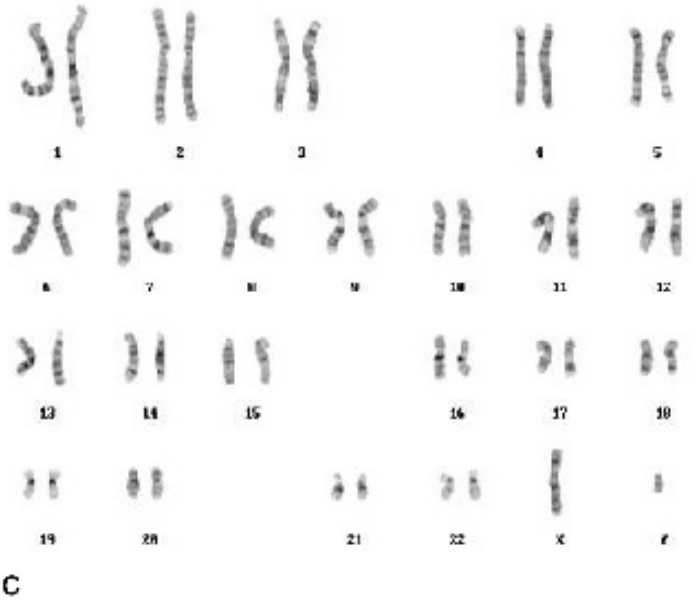

b

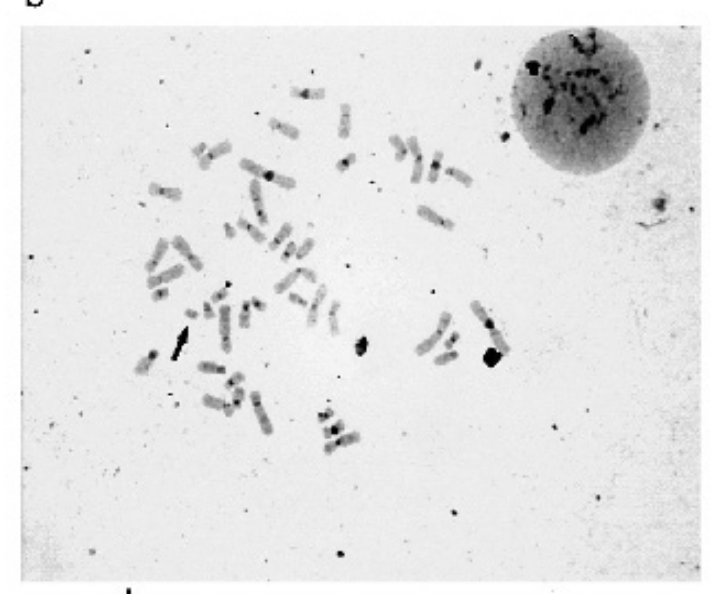

d

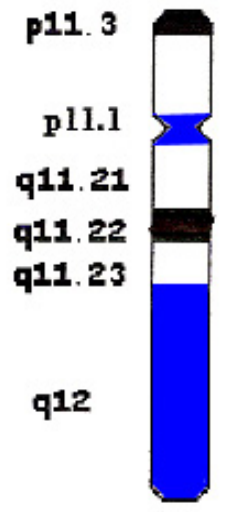

Figure 1: Cytogenetics

a) GTG Karyotype: 45,X[18]/46,XY,idic(Y)q(11.2)[82]. The Y chromosome, when present, appeared smaller than normal

b) CBG banding; absence of the heterochrmatic region on the long arm of $Y$ chromosome

c) QFQ banding by fluorescent microscopy revealed the absence of the heterochromatic region on the long arm of Y chromosome

d) Idiogram: Chromosome $\operatorname{idic}(\mathrm{Y}) \mathrm{q}(11.2)$

The use of the Alpha satellite probe, specific for a region including the centromere and spanning from Yp11.1 to Yq11, revealed the presence of 2 centromeric structures within the Y chromosome. When FISH was performed using a LSI SRY probe, specific for the SRY gene (Yp11.3), two signals were detected suggesting that the rearranged Y chromosome contained 2 copies of the SRY gene and 2 short arms. The observation was confirmed by FISH analysis using specific probes for the telomeric regions of the $\mathrm{Y}$ chromosome. In presence of the Yp specific probes, two signals corresponding to the telomeric regions of the short arms of the $Y$ chromosome were detected (Figure 2). In the same field $1 \mathrm{p}$ (green) and 1q (red) specific probes were used to detect the telomeric regions of chromosome 1 as internal control. In this context, the telomeric regions of the long arm of the Y chromosome could not be detected by Yq specific probes (green). In the same field 2p (green) and 2q (red) as well as Xq (red) specific probes were used to detect the corresponding telomeric regions of chromosomes 2 and $\mathrm{X}$, as internal control. Therefore we went more in depth with an analysis of the microdeletions in the Y chromosome via PCR. Genomic DNA was isolated by a Genomic DNA Isolation Kit (Promega, USA) using the manufacturer's protocol. The DNA sample was processed for Yq microdeletions analysis using PCR. The samples were analyzed using 12 sets of primers. In each reaction, DNA from fertile male subjects was taken as positive control and DNA from a female subject as negative control. The STS used were the following: AZF1: sY84, sY86, AZF2: sY127, sY134 (former AZFb region), sY254, sY255 (former AZFc region). ZFX and ZFY regions 2 used as internal controls. The samples were then subjected to PCR amplification. The PCR products were analyzed on a 2\% agarose gel. An STS or gene was considered absent only after at least three amplification failures in presence of both internal control (ZFY) and positive control. The test demonstrated the deletion of the STS AZF2: sY127 (former AZFb), sY254 (former AZFc) (Figure 3, lane 3) AZF2: sY134 (former AZFb), sY255 (former AZFc), (Figure 3, lane 4) whereas the STS AZF1: sY84, sY86 were present (Figure 3, lanes 3 and 4). 

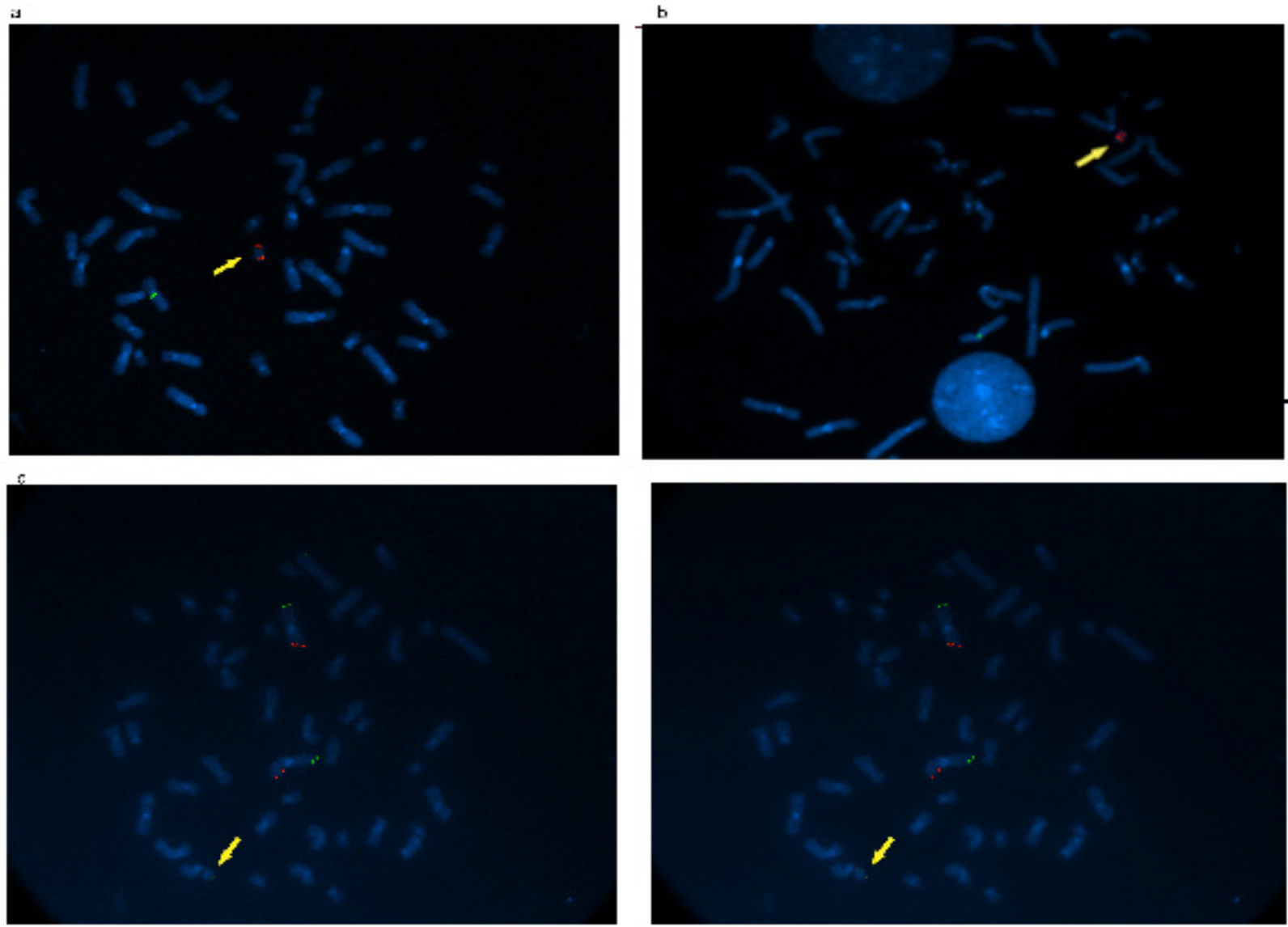

Figure 2

a) The use of the Alpha satellite probe, specific for a region including the centromere and spanning from Yp11.1 to Y q11, revealed the presence of 2 centromeric structures within the $\mathrm{Y}$ chromosome

b) FISH performed by means of a LSI SRY probe, specific for the SRY gene (Yp 11.3), two signals were detected suggesting that the re-arranged Y chromosome contained 2 SRY genes and 2 shot arms

c) In the presence of Yp specific FISH probes, two signals corresponding to the telomeric regions of the short arms of the $\mathrm{Y}$ chromosome were detected (green); in the same field $1 \mathrm{p}$ (green) and 1q (red) specific probes were used to detect the telomeric regions of chromosome 1 as internal control

d) The telomeric regions of the long arm of the $\mathrm{Y}$ chromosome could not be detected by Yq specific FISH probes (green). In the same field $2 \mathrm{p}$ (green) and $2 \mathrm{q}$ (red) as well as $\mathrm{Xq}$ (red) specific probes were used to detect the corresponding telomeric regions of chromosome 2 and $\mathrm{X}$, as internal control

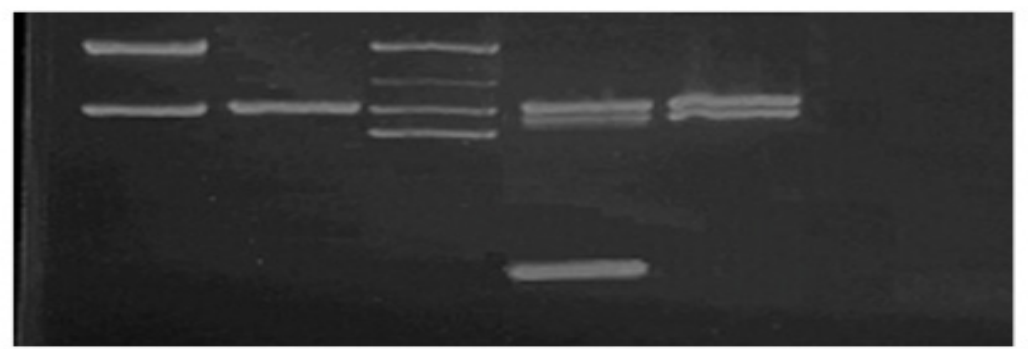

Mix A

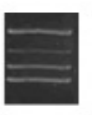

ZFXIZFY

SY 254 aziC

SY 127 azf B
Mix B
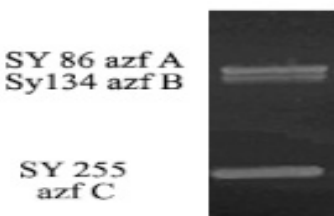

Figure 3: Analysis of Y chromosome microdeletions by PCR. The test demonstrated the deletion of the STS AZFb: sY127, AZFc: sY254 (lane 3, see Mix 1 for details); AZFb: sY134, AZFc: sY255 (lane 4, see Mix B for details); whereas the STS AZFa: sY84, sY86 were present (lanes 3 and 4). Lanes 1,2, and 5 are controls 


\section{Discussion and conclusions}

Clinical classification of Y chromosome infertility has been discussed in the introduction [1] as well as the role of molecular biology alongside cytogenetics in clinical evaluation [2]. Structural abnormalities of the Y chromosome include rings, deletions, inversions and dicentrics [5,8]. These types of abnormalities are common in infertile males, especially those with azoospermia [9]. IdicYs are mitotically unstable [10] and are consequentially associated with "sex reversal" and Turner syndrome. This is the 5th case [11] and pubmed manual search) described in the literature where this karyotype associates with "normal gross male phenotype": regular facies, non dysmorphic body and external genitalia, only microscopical alterations (sperm count and testes histology) [1214]. It's interesting that Quilter [14] also describes a case of cryptorchidism associated to azoospermia with the same karyotype. A hypothesis has also been explored, in which homologous recombination between opposing arms of MSY palindromes could generate idicY chromosomes [15]. Phenotype/karyotype correlations of Y isodicentric chromosomes have been investigated by many authors, including Hsu [5], Quilter [14], and Banks [16], along with Shinawi [17] and Al Achwar [18]. All of them made very pertinent observations about cases of mosaicism. Beaulieu Bergeron [10] also demonstrates that patients with an intercentromeric distance greater than $20 \mathrm{Mb}$ on their idic(Y) are at increased risk of having a female sexual phenotype. This report describes a subject that revealed to be a mosaic characterized by the karyotype: mos 45,X[18]/46,XY,idic(Y) $q(11.2)$ [82]. In this case, the $\mathrm{Y}$ chromosome from the second cell line is isodicentric and characterized by deletion within the long arm starting from q11.2 region; it is also characterized by the absence of the two regions of AZF2, the one within the 11.22 band and the other within the 11.23 band (former AZFb and AZFb loci), whereas the region AZF1 (former AZFa) appears to be conserved. FISH analysis displays a complex rearrangement in cell line 46, XY of the Y chromosome. The chromosome is shown to have 2 centromeres, 2 short arms with the presence of the SRY gene, the deletion of q11.23 (AZF2), part of q11.22 (AZF2), and q12. The sequences of the subtelomeric short arms are present and those of the long arm are absent. The Y chromosome is therefore constituted as described in the idiogram (Figure 1). The patient's azoospermia could be attributed to the presence of microdeletions, in particular of the DAZ (Yq11.223) gene and AZF2 gene.

Recently Kalantar et al [19] have retrospectively reviewed 14 patients with an Y (idic) chromosome, pooling together male and female phenotypes with a phenotype description that cannot be so deep, given the variety of the conditions included.

The present paper integrates and confirms previous observations, integrating the cytogenetic finding with with an accurate phenotypic description of the case.

\section{Acknowledgement}

We wish to thank A. Lindman; Dr. C. Settanni; and Dr. J. Toaff for manuscript editing and reviewing. E Colao and MFM Vismara equally contributed to this work.

\section{References}

1. Silber SJ, Disteche CM (2013) Y Chromosome Infertility, University of Washington, United States.

2. Choi DK, Gong IH, Hwang JH, Oh JJ, Hong JY (2013) Detection of Y Chromosome Microdeletion is Valuable in the Treatment of Patients With Nonobstructive Azoospermia and Oligoasthenoteratozoospermia: Sperm Retrieval Rate and Birth Rate. Korean J Urol 54: 111-6.

3. Foresta C, Moro E, Ferlin A (2001) Y chromosome microdeletions and alterations of spermatogenesis. Endocr Rev 22: 226-39.

4. Hucklenbroich K, Gromoll J, Heinrich M, Hohoff C, Nieschlag E, et al. (2005) Partial deletions in the AZFc region of the Y chromosome occur in men with impaired as well as normal spermatogenesis. Human Reprod 20: 191-7.

5. Hsu LY (1994) Phenotype/karyotype correlations of Y chromosome aneuploidy with emphasis on structural aberrations in postnatally diagnosed cases. Am J Med Genet 53: 108-40.

6. Hughes JF, Rozen S (2012) Genomics and genetics of human and primate y chromosomes. Annu Rev Genomics Hum Genet 13: 83-108.

7. Luetjens CM, Gromoll J, Engelhardt M, Von Eckardstein S, Bergmann M, et al. (2002) Manifestation of Y-chromosomal deletions in the human testis: a morphometrical and immunohistochemical evaluation. Hum Reprod 17: 2258-66.

8. Tuck-Muller CM, Chen H, Martínez JE, Shen CC, Li S, et al. (1995) Isodicentric Y chromosome: cytogenetic, molecular and clinical studies and review of the literature. Hum Genet 96: 119-29.

9. Bourrouillou G, Dastugue N, Colombies P (1985) Chromosome studies in 952 infertile males with a sperm count below 10 million/ml. Hum Genet 71: $366-7$.

10. Beaulieu Bergeron M, Brochu P, Lemyre E, Lemieux N (2011) Correlation of intercentromeric distance, mosaicism, and sexual phenotype: molecular localization of breakpoints in isodicentric Y chromosomes. Am J Med Genet A 155: 2705-12.

11. Faure AK, Aknin-Seifer I, Satre V, Amblard F, Devillard F, et al. (2007) Fine mapping of re-arranged Y chromosome in three infertile patients with nonobstructive azoospermia/cryptozoospermia. Hum Reprod 22: 1854-60.

12. Siffroi JP, Le Bourhis C, Krausz C, Barbaux S, Quintana-Murci L, et al. (2000) Sex chromosome mosaicism in males carrying Y chromosome long arm deletions. Hum Reprod 15: 2559-62.

13. Hernando C, Carrera M, Ribas I, Parear N, Baraibar R, et al. (2002) Prenatal and postnatal characterization of Y chromosome structural anomalies by molecular cytogenetic analysis. Prenat Diagn 22: 802-5.

14. Quilter CR, Nathwani N, Conway GS, Stanhope R, Ralph D, et al. (2002) A comparative study between infertile males and patients with Turner syndrome to determine the influence of sex chromosome mosaicism and the breakpoints of structurally abnormal Y chromosomes on phenotypic sex. J Med Genet 39: e80.

15. Lange J, Skaletsky H, van Daalen SK, Embry SL, Korver CM, et al. (2009) Isodicentric Y chromosomes and sex disorders as byproducts of homologous recombination that maintains palindromes. Cell 138: 855-69.

16. Banks NK, Brown L, Hughes J, Solomon B, Muenke M, et al. (2013) Phenotypic diversity amongst patients with an isodicentric y chromosome. Fertility and sterility 10: S445. 
17. Shinawi M, Cain MP, Vanderbrink BA, Grignon DJ, Mensing D, et al. (2010) Mixed gonadal dysgenesis in a child with isodicentric Y chromosome: Does the relative proportion of the 45,X line really matter? Am J Med Genet A 152A: 1832-7.

18. Al-Achkar W, Wafa A, Liehr T, Klein E, Moassass F (2012) Detailed analysis of an idic(Y) (q11.21) in a mosaic karyotype. Mol Med Rep 6: 293-6.

19. Kalantari H, Asia S, Totonchi M, Vazirinasab H, Mansouri Z, et al. (2014) Delineating the association between isodicentric chromosome Y and infertility: a retrospective study. Fertil Steril 101: 1091-6.

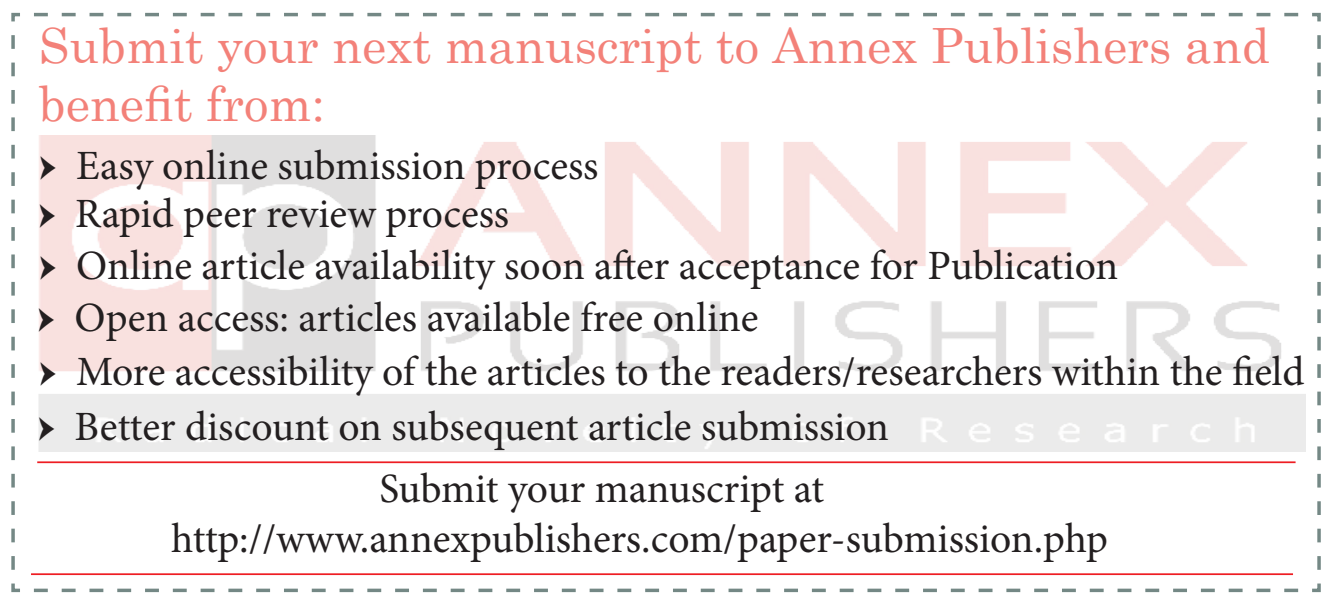

